\title{
Using Learning Analytics to Devise Interactive Personalised Nudges for Active Video Watching
}

\author{
Vania Dimitrova \\ University of Leeds, UK \\ v.g.dimitrova@leeds.ac.uk
}

\author{
Antonija Mitrovic \\ University of Canterbury, NZ \\ tanja.mitrovic@canterbury.ac.nz
}

\author{
Alicja Piotrkowicz \\ University of Leeds, UK \\ scap @leeds.ac.uk
}

\author{
Lydia Lau \\ University of Leeds, UK \\ l.m.s.lau@leeds.ac.uk
}

\author{
Amali Weerasinghe \\ University of Adelaide, Australia \\ amali.weerasinghe@adelaide.edu.au
}

\begin{abstract}
Videos can be a powerful medium for acquiring soft skills, where learning requires contextualisation in personal experience and ability to see different perspectives. However, to learn effectively while watching videos, students need to actively engage with video content. We implemented interactive notetaking during video watching in an active video watching system (AVW) as a means to encourage engagement. This paper proposes a systematic approach to utilise learning analytics for the introduction of adaptive intervention - a choice architecture for personalised nudges in the AVW to extend learning. A user study was conducted and used as an illustration. By characterising clusters derived from user profiles, we identify different styles of engagement, such as parochial learning, habitual video watching, and self-regulated learning (which is the target ideal behaviour). To find opportunities for interventions, interaction traces in the AVW were used to identify video intervals with high user interest and relevant behaviour patterns that indicate when nudges may be triggered. A prediction model was developed to identify comments that are likely to have high social value, and can be used as examples in nudges. A framework for interactive personalised nudges was then conceptualised for the case study.
\end{abstract}

\section{CCS Concepts}

- Applied Computing $\rightarrow$ Education $\rightarrow$ Interactive learning environments

\section{Keywords}

Video-based learning; soft-skills; personalised nudges, analytics.

\section{INTRODUCTION}

Video-based learning [49] is widely used in both formal education and informal learning in a variety of contexts, such as MOOCs $[18,47]$, flipped classroom [27] and problem-based learning [21]. The plethora of video content that is shared in social media platforms provides easily accessible materials for learning and teaching. Video sharing site usage has increased more than double from 2006 to 2013 [39]. On YouTube alone, 300 hours of video is uploaded every minute and almost 5 billion videos are watched

This work is licensed under a Creative Commons

UMAP '17, July 09-12, 2017, Bratislava, Slovakia. (c) 2017 Copyright is held by the owner/author(s). ACM ISBN 978-1-4503-4635-1/17/07.

http://dx.doi.org/10.1145/3079628.3079683 every day (statisticbrain.com/youtube-statistics). This creates enormous opportunities for using videos for learning in a broad range of domains. Using videos is especially powerful for soft skills learning $[12,13]$, where contextualization in personal experience and ability to see different perspectives are crucial (e.g. communicating, negotiating, collaborating). Moreover, videobased learning is seen as one of the main strategies to provide engaging learning environments for the millennials [2] who are a major target cohort for soft skills learning.

However, watching videos is inherently a passive form of learning. Numerous studies have shown that students have to actively engage with video content to learn effectively [10-13,25,38,49]. Embedding interactive activities, e.g. quizzes or assessment problems, in the videos have proven successful for engagement $[17,24,26,48]$, but these require substantial effort from the teacher and are hard to reuse. Collaborative annotation and interactive note taking [10] provide alternatives to engage learners, which require less effort from the educators and enable reuse of content.

Our research adopts interactive note-taking in video watching for soft skill learning. Previous studies found that the approach is effective only when the students actively engage with video content [34] and requirements are gathered for interactive personalised nudges to promote desired learning behaviour [35]: assist students noticing important points in videos, linking video snippets to aspects related to soft skill learning (e.g. recognise key skill elements, contextualise in past and future experience) and broadening soft skill learning portfolio (e.g. notice a variety of skill elements and use various reflection triggers when making notes).

The research presented here aims at developing a systematic approach to design interactive personalised nudges for active video watching - a novel approach that utilises analytics to derive personalisation features for extending a video-based learning environment. Both explicit user profiles (from questionnaires) and interaction traces (from system logs) are used to: (i) characterise student engagement in video-based learning, (ii) identify when interventions can be made, and (iii) predict what comments by other people may be useful as examples in the nudges. The outcome will feed into a unique choice-architecture-driven framework for the design of interactive personalised nudges.

\subsection{Active Video Watching Approach}

The active video watching approach taps into students' experiences with social media sites for video sharing (e.g. YouTube) and integrates interactive notetaking during video watching to facilitate student engagement and reflective learning. The approach is illustrated with the Active Video Watching (AVW) system [34]. In AVW, the teacher selects a set of videos for a class and defines aspects to serve as mini-scaffolds for 
learning. Aspects aim to draw the student's attention to specific points related to the target soft skill and to trigger reflective experiential learning. This paper uses an AVW instantiation for pitch presentations, which includes four YouTube video tutorials on giving presentations and four examples of pitch presentations. The following aspects are provided to stimulate recall and reflection on students' own experiences - in tutorials: "I am rather good at this", "I did/saw this in the past", "I didn't realize I wasn't doing it" and "I like this point"; in example videos, the aspects provided correspond to concepts covered in the tutorials: "Delivery", "Speech", "Structure" and "Visual aids".

Initially students watch and comment on videos individually in the PersonalSpace, using the aspects to tag their comments (Fig. 1). To enter a comment, student stops the video, types in their thoughts and selects an aspect. The system records the comment and the time elapsed from the start of the video. Once the teacher approves comments for sharing, anonymised comments are available for browsing in the SocialSpace. A second level of miniscaffolds is provided where the students are encouraged to rate the comments. The ratings, which are designed to further promote reflection, are: "This is useful for me", "I hadn't thought of this", "I didn't notice this", "I don't agree with this", and "I like this point".

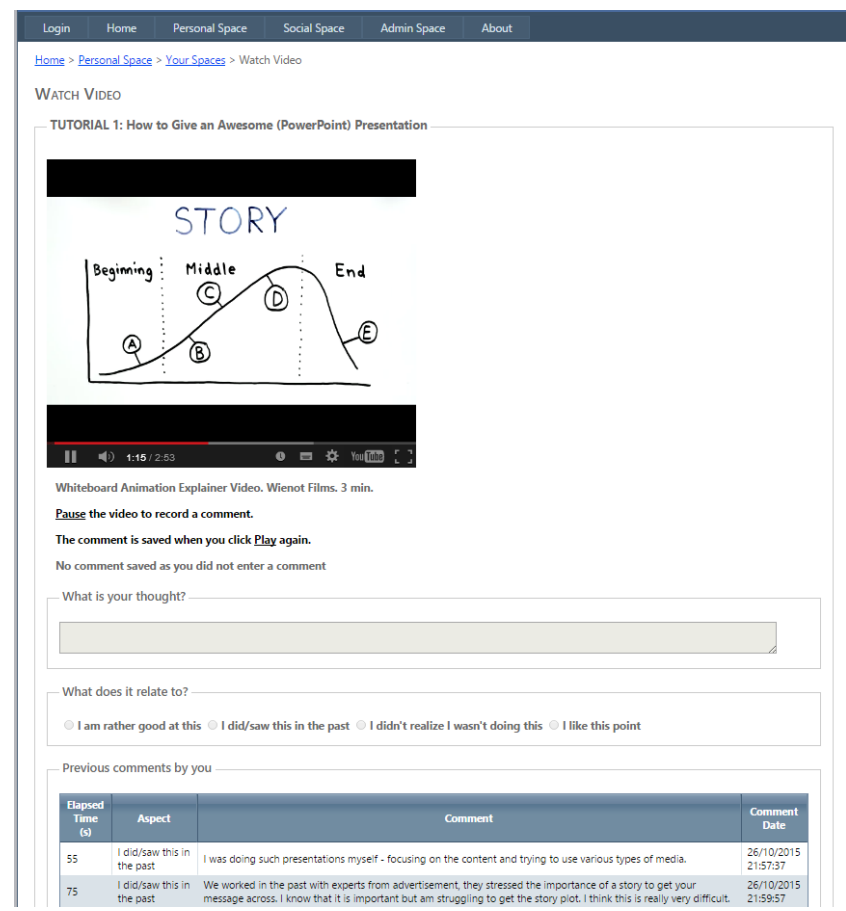

Figure 1: AVW PersonalSpace - watching a tutorial.

Two AVW studies were conducted in March and July 2016 with postgraduates and undergraduates respectively, focusing on pitch presentations [34,35]. The studies examined whether learning was happening and what kind of interactions contributed to learning. The findings showed that participants who engaged in constructive learning (i.e. wrote comments in the PersonalSpace and rated comments in the SocialSpace) improved their conceptual understanding of presentation skills, while minimal improvement for those who did not. Hence, further extension of AVW with appropriate interventions to encourage effective engagement with videos is needed. This is the aim of the research presented here.

\subsection{Nudges and Choice Architecture}

To promote engagement with videos that leads to better learning, while at the same time preserving the learners' freedom to interact with videos in a way they prefer (as common in social media platforms), we propose the use of intelligent nudges.

Nudges were introduced in decision support [46] as a form of interventions which influence people's behaviour to make choices that lead to better lives (paternalism) but in an unobtrusive and non-compulsory manner (libertarian). Behaviour change is complex and so are the corresponding interventions. Choice architecture, which defines the ways to select and present choices that can lead to better behaviour, is the core when designing nudges $[36,46]$.

To design a choice architecture for AVW nudges, we follow the development process proposed in Münscher et al. [36] and utilise learning analytics. The key principles of behaviour change [33] are noted: (i) maximise capability to regulate own behaviour; (ii) increase/reduce motivation to engage /discontinue in the desired/undesired behaviour; (iii) maximise opportunity to support self-regulation. These principles are adopted for the AVW choice architecture in the following way:

- capability: take into account both the learner's self-regulation capabilities and their knowledge /experience of the soft skill;

- motivation: aim to increase the learner's motivation to engage in active video watching and to improve their knowledge;

- opportunity: automatically identify opportunities to support engagement in active video watching to improve learning.

\subsection{Structure of Paper}

Section 2 positions the work in relevant literature. Section 3 outlines the AVW study that collected the data for analytics. Sections 4 to 6 illustrate an implementation of the proposed approach: the use of clustering techniques to understand engagement behaviours - problems/targets (S.4); a crowdwisdominspired method for identifying opportunities for intervention: video comments are analysed to identify the timing to trigger interventions and suitable example comments for sharing (S.5); a framework for designing interactive personalised nudges (S.6). The paper concludes with the main contribution and future work.

\section{RELATED WORK}

Nudges have been used in social science for interventions in lifestyle and to influence choice [46]. While sharing many features with persuasion [31], nudge is more about behaviour changes while persuasion focuses on changing beliefs. Nudge is also adopted in educational systems, including both signposting and interactive interventions. Although not explicitly called nudges, open student models can act as signposting nudges to promote reflection and self-awareness [7,27,29], with open social student models promote social comparisons [6]. These are static intervention approaches which focus on the design of effective visualisations, and rely on the students' abilities to interpret such visualisations. On the other hand, interactive intervention approaches rely on the system automatically triggers short dialogue scripts to nudge the learners to the desired behaviour. Interactive nudges can be simple reminders of college tasks [8], prompts for goal setting and reflection [27] or for navigation support [45], and dialogue games for reflection [15] or for articulation of thoughts [41]. Our approach uses interactive scripts combined with social comparison inspired by open social student modelling. We provide a unique choice-architecture-driven framework to guide the provision of suitable personalised nudges to promote video engagement for learning. Choice architecture has recently been suggested as foundational for the design of personalised interactive systems [22], though primarily used in recommender systems such as e-commerce or tourism [5]. This 
work is the first attempt to devise choice architecture for personalised nudges to improve learning.

Our approach uses learning analytics to inform the design of personalization nudges in this case by exploiting machine learning methods for clustering and classification. Clustering algorithms are commonly used to design user-adaptive systems by identifying stereotypes of users $[16,19,32,37]$. In educational settings where a range of individual differences and interaction parameters have to be considered, stereotyping require a large corpus of data. Instead, we adopt a stereotype-inspired approach that uses clusters and statistical analysis to identify problem behaviour, target behaviour and bottlenecks for not achieving the target behavior. This is combined with the analysis of interaction behaviour by other users (as in open social student models) to identify areas in a video when interactive interventions may be appropriate.

We use classification methods to predict whether comments can attract people's attention and can be used as examples to trigger learning. This builds on considerable research in using text (and features extracted from text) to predict the popularity of content $[3,4,30,43]$. The target measures to predict differ from number of comments [44] to page views or social media reactions [1]. Predictions are useful to identify items of 'good' quality content that can be used in recommendations, e.g. to automatically select the most interesting social media messages to show to a reader of a news article [42]. Similarly, we use features extracted from text and the user profile to predict 'good' quality comments. Our prediction model infers whether a given comment is of high social value, i.e. can trigger reflective learning or can induce opinion. The feature engineering and the findings of the prediction offer useful insights for researchers willing to exploit social content to enhance learning.

\section{EXPERIMENTAL SET UP}

An AVW user study was conducted in March 2016 to inform the design of personalised nudges. The goal was to investigate whether AVW was effective in supporting engagement and reflection, as well as to identify problem behaviours and what support may be provided. The participants were postgraduate students recruited through invitation sent to several mailing lists. Participation was on a voluntarily basis, including a prize draw for $\$ 100$ vouchers.

Method. The study had two phases, each one week long.

Phase 1: (PersonalSpace) After informed consent, the participants completed Survey I (collecting user profiles such as demographic information, background experiences, motivation and attitudes, and their conceptual knowledge /key concepts related to pitch presentation). The participants then received instructions on the use of AVW PersonalSpace, and advice on watching tutorials before examples. There were no further instructions as we aimed to provide an ecologically valid data collection approach which mimicked closely informal learning through video watching in YouTube. At the end of Phase 1, we administered Survey II to retest conceptual knowledge; to identify cognitive load using NASATLX [20]; and to check the perceived usefulness of the PersonalSpace using Technology Acceptance Model (TAM) [14].

Phase 2: (SocialSpace) The participants used the AVW SocialSpace to explore and rate the comments made by the others. At the end of week 2, we administered Survey III which was the same as Survey II but applied to the AVW SocialSpace.

Data logging. AVW logged the temporal data on user interactions. In this paper, we primarily use the interaction logs in the PersonalSpace and the data collected with Surveys I and II.
User ratings provided in the SocialSpace are used for identifying comments which are valuable to other learners.

Participants. 48 participants completed the profile survey. Survey II was completed by 41 participants, some of whom did not make any comments in the PersonalSpace. Since the goal of this paper is to investigate participants' engagement, we report here the $\mathbf{3 8}$ participants (26 females and 12 males) who made comments in Phase 1 and completed Surveys I and II. 17 participants were younger than 30, with the biggest group (14 participants) being aged 24-29. 6 participants were 48 or older. English was the first language for 23 participants, while the first languages of the remaining 15 participants included various Asian and European languages. 28 participants were $\mathrm{PhD}$ students. No difference between males and females, or between younger and more mature students, for prior training received on presentation skills, but there was a significant difference between native $(2,48, \mathrm{sd}=.99)$ and non-native English speakers $(1.67, \mathrm{sd}=.62)(\mathrm{U}=210.5, \mathrm{p}=$ .014). There were no significant differences on how much experience the participants had on giving presentations, how often they watched YouTube videos, or used YouTube for learning for any categories.

\section{Table 1: Summary of the MSLQ questions}

\begin{tabular}{|l|l|l|l|}
\hline & All (38) & Female (26) & \multicolumn{1}{|c|}{ Male (12) } \\
\hline Academic Control & $3.91(.46)$ & $3.96(.46)$ & $3.79(.45)$ \\
\hline Self Efficacy & $3.72(.56)$ & $3.65(.57)$ & $3.89(.51)$ \\
\hline Task Value & $4.49(.38)$ & $4.58(.33)$ & $4.31(.44)$ \\
\hline Intrinsic Motivation & $4.05(.52)$ & $4.1(.51)$ & $3.96(.56)$ \\
\hline Extrinsic Motivation & $3.37(.83)$ & $3.28(.74)$ & $3.58(1)$ \\
\hline Effort & $2.93(.44)$ & $2.9(.46)$ & $2.98(.39)$ \\
\hline Elaboration & $4.13(.54)$ & $4.15(.57)$ & $4.08(.5)$ \\
\hline Rehearsal & $3.4(.8)$ & $3.29(.79)$ & $3.66(.81)$ \\
\hline Organisation & $3.84(.94)$ & $3.94(.99)$ & $3.63(.8)$ \\
\hline Self-regulation & $3.61(.39)$ & $3.52(.36)$ & $3.8(.38)$ \\
\hline
\end{tabular}

Survey I also contained the questions from the Motivated Strategies for Learning Questionnaire (MSLQ) [40]. There were 46 questions, with the Likert scale of 1 (Not at all true of me) to 5 (Very true of me). The responses to MSLQ questions are summarized into ten dimensions in Table 1. The participants scored in the upper half of the scale on all dimensions, which is not surprising given that our participants were postgraduate students. The only marginally significant difference between male/female students is for Task Value $(U=98, p=.07)$, in which female scores higher.

AVW interaction overview. An initial statistical analysis of the collected data shows that the participants made 744 comments in the PersonalSpace, with the average of 19.58 comments per video $(s d=13.19)$. There was no significant difference between the number of comments on tutorials and examples across different demographic categories (gender, age, native/non-native speaker). Table 2 presents the distribution of comments over various aspects. The participants could make a comment without selecting an aspect, and that happened more often for tutorials (TA5) than for examples (EA5). For the four examples, comments are almost equally distributed over the given aspects, showing that the participants were watching the videos with those aspects in mind.

Survey II contained TAM questions, the replies to which were on the Likert scale from 1 (extremely likely) to 7 (extremely unlikely). The average score for usefulness of PersonalSpace (based on five questions) was 2.46 (sd=1.09), showing acceptable use. The participants answered the NASA-TLX questions on the cognitive workload imposed by the PersonalSpace using the Likert scale from 1 (Low) to 20 (High). The participants found: (i) commenting 
on the videos moderately demanding (mean $=9.89$, sd=4.87); (ii) watching and commenting on videos relatively challenging, with the average score $8.55(\mathrm{sd}=4.21)$, and there was strong positive correlation between Demand and Effort $(\mathrm{r}=.539, \mathrm{p}<.001)$; (iii) regarding whether they felt discouraged, irritated, stressed or annoyed when watching and commenting on the videos, the average score was $5.79 \quad(\mathrm{sd}=4.48)$; (iv) the self-perceived performance in identifying useful points about presentation skills has the mean score of $12.76(\mathrm{sd}=4.48)$. The distribution of scores for Performance was significantly different $(U=229, p=.02)$ for female and male participants, with male participants reporting higher values; no other significant differences between categories.

Table 2: Distribution of comments over aspects (TA tutorial aspect, EA - example aspect)

\begin{tabular}{|l|c|c|}
\hline Aspects & Comment & Ratings \\
\hline TA1: I like this point & 171 & 639 \\
\hline TA2: I didn't realize I wasn't doing this & 50 & 166 \\
\hline TA3: I am rather good at this & 33 & 128 \\
\hline TA4: I did/saw this in the past & 52 & 249 \\
\hline TA5: No aspect selected & 103 & 382 \\
\hline EA1: Delivery & 81 & 224 \\
\hline EA2: Speech & 67 & 194 \\
\hline EA3: Structure & 68 & 218 \\
\hline EA4: Visual aids & 61 & 176 \\
\hline EA5: No aspect selected & 58 & 202 \\
\hline
\end{tabular}

Conceptual knowledge of presentation skills. The change in conceptual knowledge between surveys was used as an indicator of learning. Each participant had one minute per question in the survey to write phrases they associated with (i) structure, (ii) delivery and speech, and (iii) visual aids. We developed an ontology of presentations, consisting of three taxonomies related to these areas. The answers were marked by three independent markers, indicating the number of ontology entities found with each response. The inter-rater reliability was high: the Krippendorff's alpha was 0.894 . The final scores were finalised by a fourth marker using the majority vote, or if impossible, extra marking.

The average score for conceptual knowledge from Survey I (CK1) was 12.89 (sd=6.44); Survey II (CK2) was 13.74 (6.46); Survey III (CK3) was 15.86 (6.18). Repeated measures ANOVA on the conceptual knowledge scores for the study revealed a significant effect overall $(\mathrm{F}(2,68)=6.18, \mathrm{p}=.003)$ with the partial eta squared of 0.15 (medium effect). The pairwise comparison shows there was a significant increase from Survey 1 to Survey $3(\mathrm{p}=.01)$.

In summary, we found evidence of learning, but have not found significant differences on previous experience, motivation for learning, or engagement levels between various categories of participants (e.g. age, gender). A possible explanation may be that this is a homogeneous group. However, closer examination of the data shows that there are individual differences between students on how many comments they made, and the social value of their comments. Hence, we investigate if a combination of factors can be used to discover behavioural patterns.

\section{CHARACTERISING BEHAVIOUR}

The first step in designing the choice architecture for video engagement in AVW is to identify problem behaviour and target behaviour. We do this by using unsupervised machine learning to derive clusters for characterising engagement behaviour.

We generated clusters using the k-means algorithm in SPSS, starting with 15 standardized variables from Survey I. In each run of the algorithm, variables that were not significant were removed, resulting in the final three clusters using the following variables: experience with giving presentations (Exp), using YouTube for learning $(Y 4 L)$, initial conceptual knowledge (CK1), six MSLQ variables - self-efficacy $(S E)$, academic control $(A C)$, extrinsic motivation $(E M)$, rehearsal (Reh), self-regulation $(S R)$ and organization ( $\mathrm{Org})$. Fig. 2 illustrates the cluster centers, while Table 3 reports the significant differences between the clusters (using the 2 -sided Kruskal-Wallis test). We report pairs of clusters with a significant difference on a particular variable in the last column, with a Bonferroni correction.

Cluster $\mathrm{C} 1$ has higher numbers for comments/ratings in comparison to $\mathrm{C} 2$, but the differences are not significant. $\mathrm{C} 1$ is lowest on experience overall, and lower than $\mathrm{C} 2$ on the use of YouTube for learning. C1 has the lowest scores for self-efficacy, extrinsic motivation, rehearsal, self-regulation and organization. Generally, this group is comparatively closed-minded and we refer C1 as Parochial Learners. Surprisingly, they find AVW the most useful, yet they did not benefit that much as there was no significant improvement of their conceptual knowledge.

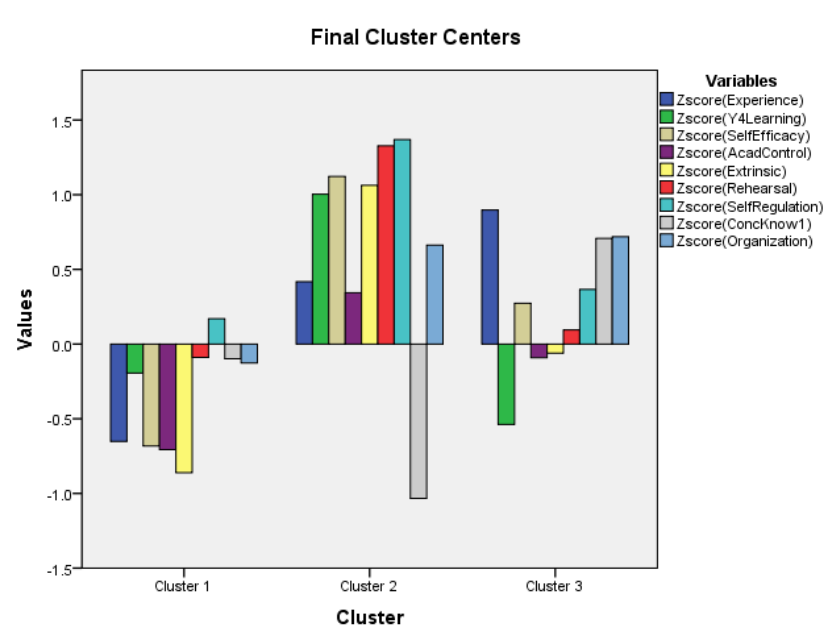

Figure 2: Cluster centres from Survey 1

The C2 participants are confident, self-regulated students but were significantly less engaged than those in cluster C3. They scored higher on extrinsic motivation, rehearsal and self-regulation. At the same time, their conceptual knowledge at the start of the study was the lowest. A possible explanation of their behaviour is that they are used to watching videos in a passive way so did not engage sufficiently. There was a marginally significant improvement on conceptual knowledge for this cluster $(\chi 2(2)=$ $5.407, \mathrm{p}=.067)$. The pairwise Wilcoxon Signed-rank test revealed a significant difference between CK1 and CK2 $(\mathrm{z}=2.003, \mathrm{p}=.045)$ and also between CK1 and CK3 $(\mathrm{z}=2.2, \mathrm{p}=.028)$. We refer to $\mathrm{C} 2$ as Habitual Video Watchers.

Cluster C3 is the "ideal" cluster illustrating the target user behaviour with AVW. The participants in this cluster were actively engaged while watching the videos, making the highest number of comments and receiving the highest number of ratings on their comments (significantly higher in comparison to $\mathrm{C} 1$ and $\mathrm{C} 2$ ). This cluster is highest on previous experience and conceptual knowledge on the pretest (CK1), and lowest on using YouTube for learning. The Friedman test revealed a significant improvement $(\chi 2(2)=6.11, \mathrm{p}=.047)$ on conceptual knowledge scores from Survey I to Survey III. The pairwise Wilcoxon Signed-rank test 
revealed a significant difference between $\mathrm{CK} 1$ and $\mathrm{CK} 3(\mathrm{z}=2.16$, $\mathrm{p}$ $=.03)$. We refer to C3 as Engaged Self-regulated Learners.

Table 3: Means (sd) for all participants and 3 clusters (C comments, $\mathrm{R}$ - Ratings, U - TAM Usefulness). * ${ }^{*}$ and * denote significance at the $0.01 / 0.05$ level respectively

\begin{tabular}{|c|c|c|c|c|c|}
\hline & $\begin{array}{l}\text { All } \\
(38)\end{array}$ & $\begin{array}{c}\text { C1 } \\
(14)\end{array}$ & $\begin{array}{l}\text { C2 } \\
\text { (7) }\end{array}$ & $\begin{array}{c}\text { C3 } \\
(17)\end{array}$ & Diff \\
\hline $\operatorname{Exp}^{* *}$ & $\begin{array}{l}2.87 \\
(.78)\end{array}$ & $\begin{array}{l}2.21 \\
(.58)\end{array}$ & $3(.58)$ & $\begin{array}{l}3.35 \\
(.61)\end{array}$ & $\begin{array}{l}\mathrm{C} 1-\mathrm{C} 2{ }^{*} \\
\mathrm{C} 1-\mathrm{C} 3\end{array}$ \\
\hline $\mathrm{Y} 4 \mathrm{~L}^{* *}$ & $\begin{array}{c}2.71 \\
(1.01)\end{array}$ & $\begin{array}{l}2.64 \\
(1.01)\end{array}$ & $3.86(.9)$ & $\begin{array}{l}2.29 \\
(.69)\end{array}$ & $\begin{array}{l}\mathrm{C} 1-\mathrm{C} 2{ }^{*} \\
\mathrm{C} 2-\mathrm{C} 3{ }^{* *}\end{array}$ \\
\hline $\mathrm{SE}^{* *}$ & $\begin{array}{l}3.73 \\
(.56)\end{array}$ & $\begin{array}{l}3.29 \\
(.45)\end{array}$ & $4.31(.4)$ & $\begin{array}{l}3.83 \\
(.41)\end{array}$ & $\begin{array}{l}\mathrm{C} 1-\mathrm{C} 2{ }^{* *} \\
\mathrm{C} 1-\mathrm{C} 3{ }^{*}\end{array}$ \\
\hline $\mathrm{EM}^{* *}$ & $\begin{array}{l}3.37 \\
(.83)\end{array}$ & $\begin{array}{l}2.84 \\
(.59)\end{array}$ & $\begin{array}{l}4.29 \\
(.34)\end{array}$ & $\begin{array}{l}3.44 \\
(.81)\end{array}$ & $\begin{array}{l}\mathrm{C} 1-\mathrm{C} 2{ }^{* *} \\
\mathrm{C} 2-\mathrm{C} 3\end{array}$ \\
\hline $\operatorname{Reh}^{* *}$ & $\begin{array}{l}3.4 \\
(.8)\end{array}$ & $\begin{array}{l}3.11 \\
(.49)\end{array}$ & $\begin{array}{l}4.32 \\
(.49)\end{array}$ & $\begin{array}{l}3.27 \\
(.85)\end{array}$ & $\begin{array}{l}\mathrm{C} 1-\mathrm{C} 2{ }^{* *} \\
\mathrm{C} 2-\mathrm{C} 3\end{array}$ \\
\hline $\mathrm{SR}^{* *}$ & $\begin{array}{l}3.61 \\
(.39)\end{array}$ & $\begin{array}{l}3.45 \\
(.31)\end{array}$ & $\begin{array}{l}4.08 \\
(.32)\end{array}$ & $\begin{array}{l}3.55 \\
(.33)\end{array}$ & $\begin{array}{l}\mathrm{C} 1-\mathrm{C} 2{ }^{* *} \\
\mathrm{C} 2-\mathrm{C} 3{ }^{*}\end{array}$ \\
\hline Org* & $\begin{array}{l}3.84 \\
(.94)\end{array}$ & $\begin{array}{l}3.25 \\
(.99)\end{array}$ & $\begin{array}{l}4.14 \\
(.75)\end{array}$ & $\begin{array}{l}4.21 \\
(.73)\end{array}$ & $\mathrm{C} 1-\mathrm{C} 3$ * \\
\hline $\mathrm{CK} 1^{* *}$ & $\begin{array}{l}12.89 \\
(6.43)\end{array}$ & $\begin{array}{l}11.86 \\
(5.16)\end{array}$ & $\begin{array}{l}6.71 \\
(5.22)\end{array}$ & $\begin{array}{l}16.29 \\
(5.83)\end{array}$ & $\mathrm{C} 2-\mathrm{C} 3{ }^{* *}$ \\
\hline $\mathrm{CK}^{*}$ & $\begin{array}{l}13.74 \\
(6.46)\end{array}$ & $\begin{array}{l}12.71 \\
(6.37)\end{array}$ & $\begin{array}{l}9.14 \\
(3.93)\end{array}$ & $\begin{array}{l}16.47 \\
(6.31)\end{array}$ & $\mathrm{C} 2-\mathrm{C} 3$ * \\
\hline $\mathrm{CK}^{*}$ & $\begin{array}{l}15.86 \\
(6.18)\end{array}$ & $\begin{array}{l}14.46 \\
(6.36)\end{array}$ & $\begin{array}{l}12 \\
(5.89)\end{array}$ & $\begin{array}{l}18.87 \\
(4.93)\end{array}$ & $\mathrm{C} 2-\mathrm{C} 3{ }^{*}$ \\
\hline $\mathrm{C}^{*}$ & $\begin{array}{c}19.58 \\
(13.19)\end{array}$ & $\begin{array}{l}18.71 \\
(14.38)\end{array}$ & $\begin{array}{l}10 \\
(7.26)\end{array}$ & $\begin{array}{l}24.24 \\
(12.27)\end{array}$ & $\mathrm{C} 2-\mathrm{C} 3{ }^{*}$ \\
\hline $\mathrm{R}^{*}$ & $\begin{array}{c}68.08 \\
(49.36)\end{array}$ & $\begin{array}{l}63.79 \\
(45.64)\end{array}$ & $\begin{array}{l}32.29 \\
(19.31)\end{array}$ & $\begin{array}{l}86.35 \\
(53.59)\end{array}$ & $\mathrm{C} 2-\mathrm{C} 3$ * \\
\hline $\mathrm{U}^{* *}$ & $\begin{array}{l}3.91 \\
(.38)\end{array}$ & $\begin{array}{l}3.65 \\
(.34)\end{array}$ & $\begin{array}{l}4.24 \\
(.37)\end{array}$ & $\begin{array}{l}3.99 \\
(.27)\end{array}$ & $\begin{array}{l}\mathrm{C} 1-\mathrm{C} 2{ }^{* *} \\
\mathrm{C} 1-\mathrm{C} 3\end{array}$ \\
\hline VTA $^{*}$ & $\begin{array}{c}2.39 \\
(1.29)\end{array}$ & $\begin{array}{l}2.21 \\
(1.05)\end{array}$ & $\begin{array}{l}1.29 \\
(1.25)\end{array}$ & $3(1.17)$ & $\mathrm{C} 2-\mathrm{C} 3$ * \\
\hline VEA $^{*}$ & $\begin{array}{c}2.63 \\
(1.72)\end{array}$ & $\begin{array}{l}3.07 \\
(1.39)\end{array}$ & $1(1.73)$ & $\begin{array}{l}2.94 \\
(1.64)\end{array}$ & $\begin{array}{l}\mathrm{C} 1-\mathrm{C} 2{ }^{*} \\
\mathrm{C} 2-\mathrm{C} 3{ }^{*}\end{array}$ \\
\hline $\mathrm{RC}^{* *}$ & $\begin{array}{c}3.5 \\
(4.21)\end{array}$ & $\begin{array}{l}2.29 \\
(2.7)\end{array}$ & $\begin{array}{l}.71 \\
(1.25)\end{array}$ & $\begin{array}{l}5.65 \\
(4.99)\end{array}$ & $\mathrm{C} 2-\mathrm{C} 3{ }^{* *}$ \\
\hline PropR $^{* *}$ & $.28(.22)$ & $.21(.17)$ & $.1(.19)$ & $.41(.21)$ & $\begin{array}{l}\mathrm{C} 1-\mathrm{C} 3{ }^{*} \\
\mathrm{C} 2-\mathrm{C} 3{ }^{* *}\end{array}$ \\
\hline
\end{tabular}

We further analysed the comments made by each cluster in terms of aspects. In Table 3, the Variety of Tutorial Aspects (VTA) and Variety for Example Aspects (VEA) are reported. The average number of distinct aspects used by the whole population for tutorials is 2.39. There was a significant difference on the average VTA scores of the three clusters $(\mathrm{H}=9.25, \mathrm{p}=.01)$, with $\mathrm{C} 3$ being significantly higher than $\mathrm{C} 2(\mathrm{p}=.01)$. Fig. 3 shows the average number of comments per tutorial aspects for the three clusters, as well as for reflective aspects (RA, which includes TA2, TA3 and TA4). There was a significant difference on the average number of reflective comments ( $\mathrm{RC}$ in Table 3$)(\mathrm{H}=11.87, \mathrm{p}=.003)$, with $\mathrm{C} 3$ making significantly more reflective comments in comparison to $\mathrm{C} 2(\mathrm{p}=.01)$. There was also a significant difference on the proportional use of reflective aspects (PropR) $(\mathrm{H}=11.78, \mathrm{p}=.003)$, with $\mathrm{C} 3$ having a significantly higher proportion in comparison to $\mathrm{C} 1(\mathrm{p}=.04)$ and $\mathrm{C} 2(\mathrm{p}=.005)$. Most of the comments the $\mathrm{C} 2$ participants made used TA1 or no aspect. There was also a significant difference between the average numbers of aspects for comments on examples $(\mathrm{H}=7.59, \mathrm{p}=.022)$, with a significant pairwise difference between $\mathrm{C} 1$ and $\mathrm{C} 2(\mathrm{p}=.04)$, and also between $\mathrm{C} 2$ and $\mathrm{C} 3(\mathrm{p}=.03)$.

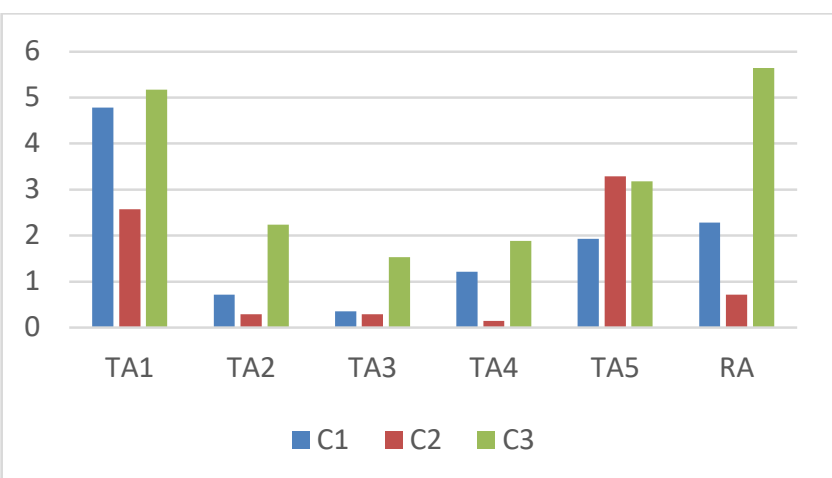

Figure 3: Average number of comments per tutorial aspect

To summarise the findings above, two main patterns of problem behaviours were identified - C1: Parochial Learners and C2: Habitual Video Watchers; with the target behaviour being C3: Engaged Self-regulated Learners. Participants in C3 had their conceptual knowledge improved significantly during the study. They made the most comments, which had the highest social value, and used reflective aspects significantly more often in comparison to $\mathrm{C} 1$ and $\mathrm{C} 2$. The participants in cluster $\mathrm{C} 1$, who did not improve their conceptual knowledge during the study, had low experience, and lacked self-regulation and learning skills. Although they commented on videos, their comments had low social value. Their SR and learning skill need to be improved. The participants in cluster C2 had strong SR and learning skills, but had lowest prior conceptual knowledge and the lowest engagement level. They need to acquire more conceptual understanding in order to recognise opportunities for commenting/rating, to be able to engage at a higher level and use a greater variety of aspects when commenting.

\section{IDENTIFYING OPPORTUNITIES FOR INTERVENTION}

To design nudges, we need to identify opportunities for intervention, i.e. to decide when there may be a suitable time for an intervention, and what to include in a nudge. For this, we propose the use of interaction traces generated by learners in the user study. We look for video intervals worthy for attention and investigate ways to identify comments for examples in the nudges.

\subsection{Attention Intervals}

An attention interval $I$ is defined as a continuous stretch of video consisting of a set of comments $C$. The granularity of continuity is determined by how big time gap $\theta$ is allowed between adjacent 
comments. We define an aggregation predicate $A(C)$, which aggregates comments from a given set $C$, as follows:

$$
A(C) \equiv \forall\left(c_{i} \in C\right) \exists\left(c_{j} \in C\right)\left[\left(c_{i} \neq c_{j}\right) \wedge \operatorname{distance}\left(c_{i}, c_{j}\right) \leq \theta\right]
$$

This allows us to aggregate comments in attention intervals that indicate areas in a video where users have noted something. Table 4 summarises the output of interval aggregation for the eight videos used in the study. The time distance parameter $\theta$ was set to $4 "$ for tutorials and 6" for examples, and was selected as the maximum number that gives a reasonable interval partitioning (larger values of $\theta$ will aggregate almost all comments in one interval).

Table 4: Summary of the interval aggregation for each video.

\begin{tabular}{|l|c|c|c|c|c|}
\hline Video & Length & $\begin{array}{c}\text { \# of } \\
\text { Comments }\end{array}$ & $\begin{array}{c}\text { \# of } \\
\text { Intervals }\end{array}$ & $\begin{array}{c}\text { Avg.Int. } \\
\text { Length }\end{array}$ & $\begin{array}{c}\text { \# Int. with } \\
\text { Length> 10" }\end{array}$ \\
\hline T1 & $2.54^{\prime}$ & 89 & 10 & $9 "(13)$ & 2 \\
\hline T2 & $7.37^{\prime}$ & 110 & 20 & $6 "(7)$ & 2 \\
\hline T3 & $6.55^{\prime}$ & 120 & 23 & $6 "(4)$ & 4 \\
\hline T4 & $6.22^{\prime}$ & 90 & 15 & $6 "(3)$ & 3 \\
\hline E1 & $3.23^{\prime}$ & 79 & 9 & $11^{\prime \prime}(7)$ & 4 \\
\hline E2 & $8.28^{\prime}$ & 93 & 19 & $7 "(4)$ & 7 \\
\hline E3 & $6.48^{\prime}$ & 100 & 20 & $9 "(6)$ & 8 \\
\hline E4 & $3.25^{\prime}$ & 63 & 7 & $15^{\prime \prime}(11)$ & 5 \\
\hline
\end{tabular}

Because the intervals vary in length, number of comments, representation of clusters and aspects, we look at ways to extract those that are useful to encourage engagement. Taking into account that the intervals can be used to direct a user to a place in the video and that he/she would have to have adequate time to absorb the point made, the interval length can be used as a filter to get useful attention intervals. For example, if we put a filter of length greater than 10", the number of attention intervals reduces noticeably, as shown in Table 4. Other ways to filter could be applied, e.g. take the $k$ longest intervals for each video.
Further processing of the comments in an interval allows us to identify useful patterns for the timing of interventions. Considering that the problem behaviour for AVW nudges is related mainly to clusters $\mathrm{C} 1$ and $\mathrm{C} 2$ and the target behaviour is related to cluster C3 (as identified in Section 3), we further analyse the behaviour of clusters with these intervals. Five patterns were identified (see illustrations in Fig. 4). When cluster C1 was the only one engaged (pattern P4), the comments referred to unimportant aspects (e.g. a comment noting that power point should be used). In such cases, the learner may be encouraged to continue to look at aspects in other intervals, such as those where many people commented and used diverse aspects (pattern P1). We noted intervals when a cluster did not engage, while the others did (patterns P2 and P5). When learners from a disengaged cluster approach such intervals, existing comments can be shown as examples of other people's opinions to direct attention to important aspects and to stimulate interaction. There were intervals where only cluster C3 (our target behaviour) engaged (pattern P3), and this indicate learning points that only people with experience in the soft skill may notice. It may be hard to stimulate learners from clusters $\mathrm{C} 1$ and $\mathrm{C} 2$ to notice these points when they lack experience; instead, once the learner is at such an interval, s/he can be encouraged to pause and read comments from more experienced people for reflection.

\subsection{Comments with Social Value}

While attention intervals and patterns can indicate when to make a nudge, comments from others can provide examples that can be used in a nudge to stimulate engagement. Not every comment will be stimulating. As a proxy for the social value of a comment, i.e. whether the comment will be of interest to others, the ratings received in the SocialSpace will be used.

Table 5 summarises the rating metrics - ratings R1-R3 Trigger Learning, as they indicate that people notice something new,
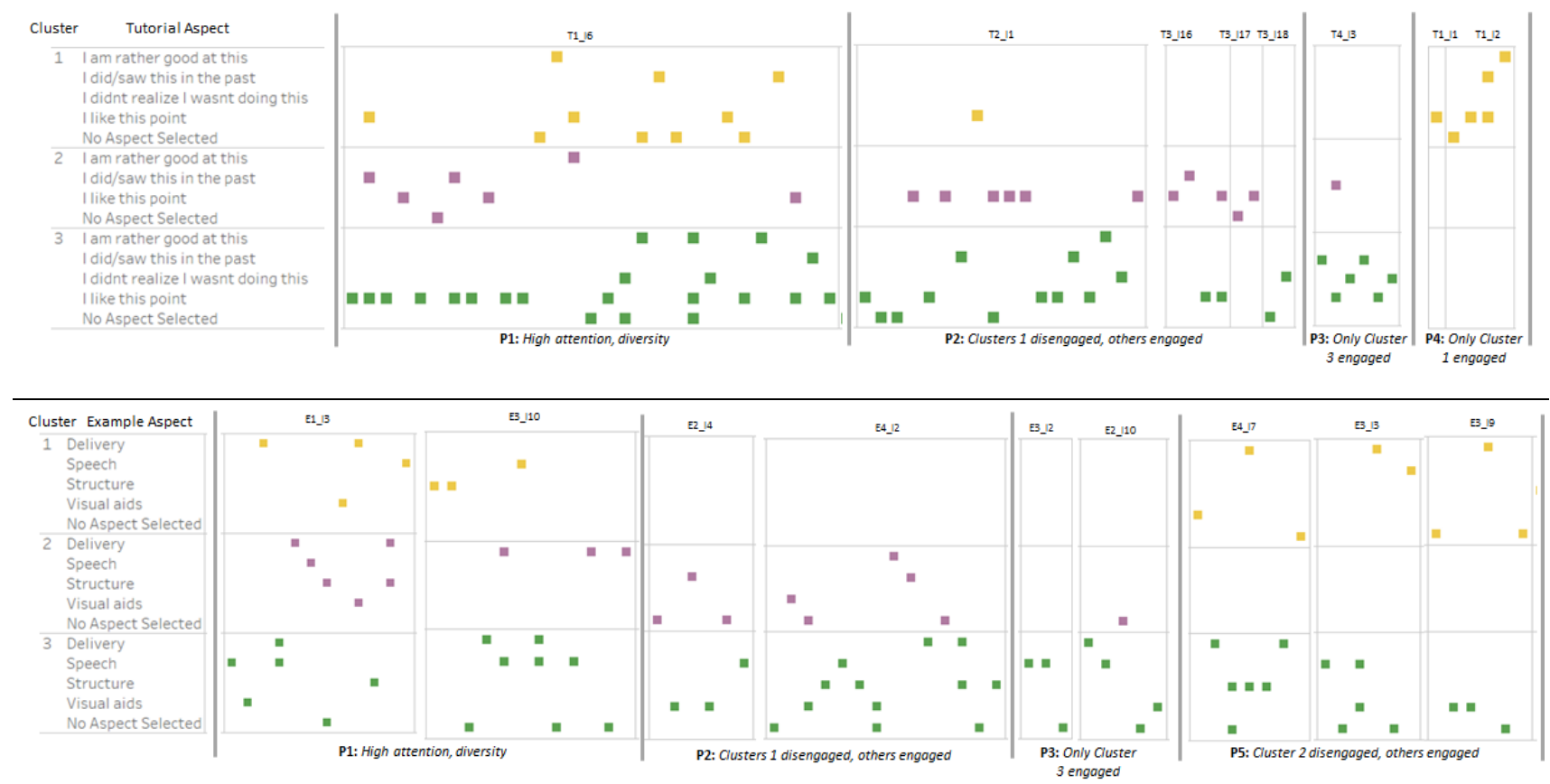

Figure 4: Interval patterns that indicate situations when interventions can be triggered. Top- intervals from tutorial videos ( ${ }_{i} \_I j$ indicates interval $j$ in tutorial $i$ ). Bottom- intervals from example videos $\left(E i_{-} I j\right.$ indicates interval $j$ in example $i$ ). 
unnoticed in their own comments; while R4-R5 Induce Opinion, as people state that they disagree or that they like the point.

Table 5: Summary of the ratings on comments in SocialSpace.

\begin{tabular}{|l|l|c|}
\hline Class & Rating category & \# Ratings \\
\hline \multirow{3}{*}{$\begin{array}{l}\text { Trigger } \\
\text { Learning }\end{array}$} & R1: This is useful for me & 349 \\
\cline { 2 - 3 } & R2: I hadn't thought of this & 260 \\
\cline { 2 - 3 } & R3: I didn't notice this & 241 \\
\hline \multirow{2}{*}{$\begin{array}{l}\text { Induce } \\
\text { Opinion }\end{array}$} & R4: I do not agree with this & 213 \\
\cline { 2 - 3 } & R5: I like this point & 1643 \\
\hline
\end{tabular}

We present a method to identify high social value comments, using linguistic and user profile features of comments, and employ natural language processing and machine learning techniques. The data set used includes 742 comments, which range in length from 1 to 97 tokens (median=10) and follow a Zipfian distribution. For calculating correlations and in the prediction model, the ratings were normalised by the total number of ratings on a given video.

Feature Engineering. In order to find high social value comments, we consider three feature groups: the comments' linguistic content, domain-specific keywords, and comment metadata (including the user profile and aspects).

Linguistic features. We used the Linguistic Inquiry and Word Count (LIWC) tool (https://liwc.wpengine.com) which analyses texts and returns the percentages of words from its topical dictionaries. These dictionaries include grammar, affect, cognitive processes, formality, and punctuation. Since comments do not always follow proper grammar, the approach using keyword counts as employed by LIWC is more appropriate than full parsing and discourse analysis. LIWC output consists of 93 features.

Domain-specific keywords. Since experience in the relevant domain could lead to writing higher social value comments, we created a lexicon of keywords that relate to various aspects of making presentations (delivery/speech, structure, visual aids). The lexicon consists of 380 single words (e.g. articulate, outline) and 61 phrases (e.g. easy to understand). We implement two features: the proportion of domain-specific keywords to all tokens in the comment text, and the conceptual knowledge terms provided by the learners in the study pre-test in Survey I (as described in Section 3).

Metadata. Comment metadata (26 features) were implemented as binary features. The metadata relates to the user profile: gender, English as native language (both implemented as binary features), self-reported experience level in this domain, engagement with the system (\# comments made, \# videos watched), user cluster (from Section 4), and results from the MSLQ (Survey I).

Notable correlations. We calculated correlations between feature values and the three target measures for example and tutorial videos separately. Kendall's tau was used for numeric features and point-biserial correlation for binary features (we report: $\left.{ }^{*} \mathrm{p}<.05,{ }^{* *} \mathrm{p}<.01,{ }^{* * *} \mathrm{p}<.001\right)$. We found approximately a third of them to have a significant correlation with at least one target measure. Significant correlations fall across all feature groups highlighting the need to consider different aspects of comments in order to predict their social value.

In general, there were noticeably more significant correlations between linguistic features and target measures for example videos, whereas for tutorial videos user profile features were significantly correlated more often. Comments made by users with a higher pre-existing knowledge of the domain were more likely to trigger learning $\left(.08^{*}\right.$ for tutorials, $.11^{* *}$ for examples). For tutorials, comments from users who are intrinsically motivated $\left(.09^{*}\right)$ and have good organization skills $\left(.11^{* *}\right)$ were more likely to trigger learning. Similarly, comments from users who engaged more by commenting on tutorials were more likely to induce opinions $\left(.08^{*}\right)$. In terms of linguistic features comments with higher proportions of personal pronouns (especially 'we') are more likely to induce opinions in example videos (personal pronouns: $.11^{* *}$, 'we': .1*). Also in example videos, there is a negative correlation between learning ratings and using words relating to negative emotion (e.g. hurt, -.16 ${ }^{* * *}$ ), anger (e.g. annoy, $-.1^{*}$ ) and risk (e.g. doubt, $\left.-.12^{*}\right)$, and a positive correlation between opinion ratings and using words relating to causation (e.g. because, . $1^{*}$ ), certainty (e.g. always, . $1^{*}$ ), and future (e.g. soon, $\left..14^{* *}\right)$. For tutorial videos comments with longer sentences (words per sentence: $.09^{* *}$ ) and with more words relating to space (e.g. down, $.1^{* *}$ ) are more likely to get opinion ratings, while a higher proportion of words relating to the body (e.g. hands, $\left.-.09^{*}\right)$ is negatively correlated with learning ratings. Comments with the aspect "I didn't realize I wasn't doing $i t$ " are more likely to trigger learning on tutorial videos $\left(.12^{*}\right)$.

Prediction Model. Our aim is to identify comments with high social value. A high social value comment is one which has a number of ratings in the top quartile for the dataset. Thus each comment is denoted as a 'Good' or 'Not Good', making this a binary classification task. In order to address the class imbalance (only approx. 22\% of the comments belong to the 'Good' class), we utilise a method called SMOTE [9], which undersamples the majority class and synthetically oversamples the minority class. Furthermore, we remove any features which have zero or nearzero variance. We tested a number of algorithms. Random Forest yielded the best results (it has been shown to work well on datasets with a mix of numeric and binary features).

Evaluation. We run a 10 -fold cross-validation and report accuracy (percentage of correctly classified instances), precision and recall. Results are reported in Table 6. Majority class baseline achieves .77 accuracy and zero precision and recall. The prediction model achieves very good performance of at least $90 \%$ accuracy for all measures, with at least .83 precision and .92 recall. This means

Table 6: Classification results.

\begin{tabular}{|l|l|l|l|l|l|l|l|l|}
\hline \multicolumn{2}{|l|}{ All Ratings } & \multicolumn{3}{|c|}{ Trigger Learning } & \multicolumn{3}{l|}{ Induce Opinion } \\
\hline Acc. & Prec. & Rec. & Acc. & Prec. & Rec. & Acc. & Prec. & Rec. \\
\hline .90 & .84 & .93 & .90 & .83 & .92 & .91 & .85 & .93 \\
\hline
\end{tabular}

that we can accurately identify comments with high social value.

Table 7: Example comments with predicted class/actual class.

(1) Important to have faith in yourself and believe that your message is important (Tutorial, Good/Good)

(2) A lot to remember, but good tips (Tutorial, Not Good/Not Good)

(3) Clear ending, the audience leaves remembering the main idea. (Example, Good/Good)

(4) confusing visual (Example, Not Good/Not Good)

(5) Speaks very quickly, comes across as passionate, but some pauses would be better (Example, Good/Not Good)

(6) speaking quickly (Example, Not Good/Good)

Overall, the output of the prediction model indicates that it is possible to identify comments with high social value. Examples (1)-(4) in Table 7 show that the model makes correct prediction across video types. Examples (5) and (6) highlight the challenging 
nature of our task. They address a similar point and even use some similar words, however (6) attracted more ratings. These similarities between comments in different classes leads to some confusion in the model. Hence, we propose that it will be more appropriate to use the 'Good' class probability as a measure for ranking comments, and this will allow to select from a pool of comments those which are of the best quality within the given set.

\section{FRAMEWORK FOR INTERACTIVE PERSONALISED NUDGES}

This section utilises the analytics presented in sections 4 and 5 to instantiate a choice-architecture-driven framework for specifying interactive personalised nudges to improve active video watching (illustrated in AVW). Following [15], we present each nudge as a dialogue game $N=\langle G, P, T, O\rangle$, where $G$ defines the goal of the game (i.e. the problem behaviour that we want to change with the nudge), $P$ defines the conditions when the game will be triggered (i.e. situation(s) when the intervention will be generated), $T$ defines the interaction template (canned text which is instantiated according to the context), and $O$ defines the expected outcome (elements of target behaviour we want to achieve with the nudge).

Context model. To enable adaptation, we propose a context model $C=<U_{\mathrm{YT}}, U_{\mathrm{K}}, U_{\mathrm{MSLQ}}, U_{\mathrm{L}}, V_{\mathrm{I}}, V_{\mathrm{C}}>$ that includes information about both the user and the video. Explicit profiling obtained before interaction with AVW (Section 3) includes $U_{\mathrm{YT}}$ (the user's experience in using YouTube videos for learning), $U_{\mathrm{K}}$ (the user's knowledge and previous experience in the target skill), and $U_{\text {MSLO }}$ (the MSLQ scales used for generating the clusters: self-efficacy, extrinsic motivation, rehearsal, self-regulation, and organization). Implicit profiling from interaction logs $U_{\mathrm{L}}$ includes the number of comments and frequencies of video aspect usage (Section 4). The video information aggregates the interaction traces by others (Section 5), including $V_{\mathrm{I}}$ (the set of high attention intervals with detected interaction patterns) and $V_{\mathrm{C}}$ (for each comment, the probability that the comment belongs to class 'Good' social value).

Nudge categories. Münscher et al. [36] aggregate the empirically tested choice architecture interventions into three nudge categories, which can be used to guide the design of AVW nudges:

Decision information nudges facilitate the perceptual processes of problem representation, formulation, or framing to help people process the available information that can affect their behavior. In AVW, this includes nudges that provide information before interacting with AVW or before entering intervals when making comments would be beneficial for learning.

Decision structure nudges facilitate assessment and selection of alternatives when a decision is to be made, including range/composition of options and default options. In AVW, this refers to nudges that help identify the appropriate aspect for a comment or show comments made by others. These nudges are made when the learner is within an attention interval and there is a learning point to be noticed and associated to an aspect.

Decision assistance nudges foster deliberate commitment and remind people of behavioral options. In AVW, this refers to nudges that provide feedback on engagement and 'reward' positive engagement behaviour. They can be triggered after an attention interval is passed or after a video has been watched.

In addition to the intervention techniques for each nudge category suggested in [36], tips for teacher interventions informed by MSLQ categories [23] are also used to devise nudges with pedagogical goals. Table 8 illustrates the three nudge types, with corresponding techniques: provide social reference point, use prompted choice, and facilitate commitment. There can be nudges with the same goal which can be triggered in different preconditions and can be implemented with different nudging techniques.

Table 8: Example dialogue games for AVW nudges.

N1: [Decision information: provide social reference point] $G$ : Direct the attention of a Parochial Learner.

$\boldsymbol{P}$ : $U_{\mathrm{K}}$ is low, $U_{\mathrm{MSLQ}}$ values are lower than collective mean, \# of comments in $U_{\mathrm{L}}$ is around the video average. The learner is approaching an attention interval with pattern P3 (only Cluster 3 engaged), it has at least one 'Good' social value comment.

T: 'You are about to watch a part where other students made comments, for example [show 'Good' social value comment].'

$\boldsymbol{O}$ : The learner makes a relevant comment.

N2: [Decision structure: use prompted choice]

$G$ : Promote engagement of a Habitual Video Watcher.

$\boldsymbol{P}$ : $U_{\mathrm{YT}}$ is high, $U_{\mathrm{K}}$ is low, $U_{\mathrm{MSLQ}}$ values are higher than the collective mean, variety of used video aspects as indicated by the aspect frequency in $U_{\mathrm{L}}$ is low, the learner is in an attention interval with pattern $P 1$ (high attention high diversity).

T: 'Have you thought about [unused aspect]. For example, somebody else has said [show 'Good' social value comment].'

$\boldsymbol{O}$ : The learner starts to relate comments to more video aspects.

N3: [Decision assistance: facilitate commitment]

$G$ : Reward positive behaviour.

$\boldsymbol{P}$ : The learner has made a comment that has a high probability to belong to 'Good' social value class.

T: 'You made a very good comment that can be useful to others [show user comment].

$\boldsymbol{O}$ : The learner's motivation and knowledge increase.

\section{CONCLUSIONS}

The work presented here contributes to an emerging research stream that exploits the synergy between the areas of learning analytics and user-adaptive systems. We pioneer a data-driven approach where insights from analytics are used to inform the provision of user-adaptive interventions in an existing system. This is illustrated with a case study that used AVW to learn about pitch presentation. Our main contribution is a systematic methodology to (i) populate a context model for learning through active video watching, which includes information about both the learner (explicit and implicit profiling) and the video (aggregating traces of user interaction with videos) and (ii) devise the choice architecture for active video watching nudges, by identifying from the analytics (a) problem behaviours and a target behaviour, (b) appropriate attention intervals and patterns for triggering nudges, and (c) comments that can be used as examples in the nudges to trigger reflective learning or to induce opinion.

Our future work will examine the effectiveness of the nudges in AVW with an experimental study, and how to provide the nudges in addition to the when and what. Transferability to other population and systems will need to be investigated.

Acknowledgements. This research was supported by the EUFP7-ICT-257184 ImREAL grant, an EPSRC Doctoral Training Grant, a teaching development grant from the University of Canterbury, and an Ako Aotearoa grant. 


\section{REFERENCES}

[1] I. Arapakis, B. B. Cambazoglu, and M. Lalmas. 2014. On the feasibility of predicting news popularity at cold start. In International Conference on Social Informatics. Springer International Publishing. 290-299.

[2] E. Arelando 2013. The Millennials Are Coming! Proven Engagement Strategies. Learning Solutions Magazine.

[3] V. G. Ashok, S. Feng, and Y. Choi. 2013. Success with style: Using writing style to predict the success of novels. Poetry, 580(9), 70.

[4] R. Bandari, S. Asur, and B.A. Huberman. 2012. The Pulse of News in Social Media: Forecasting Popularity. In ICWSM.

[5] E. Bothos, D. Apostolou, and G. Mentzas. 2015. Recommender systems for nudging commuters towards ecofriendly decisions. Intelligent Decision Technologies, 9(3), 295-306.

[6] P. Brusilovsky, S. Somyürek, J. Guerra, R. Hosseini, and V. Zadorozhny. 2015. The value of social: Comparing open student modeling and open social student modeling. Proc. Int. Conf. User Modeling, Adaptation, and Personalization. Springer International Publishing. 44-55.

[7] S. Bull, and J. Kay. 2016. SMILI(-): a framework for interfaces to learning data in Open Learner Models, learning analytics and related fields. Artificial Intelligence in Education, 26(1), 293-331.

[8] B. L. Castleman, and L. C. Page. 2013. Summer nudging: Can text messages and peer mentor outreach increase college going among low-income high school graduates. In Soc. for Research on Educational Effectiveness Spring Conf., DC.

[9] N. V. Chawla, K. W. Bowyer, L. O. Hall, and W. P. Kegelmeyer. 2002. SMOTE: synthetic minority over-sampling technique. Journal of artificial intelligence research, 16, 321357.

[10] M. A. Chatti, M. Marinov, O. Sabov, et al. (2016). Video annotation and analytics in Course-Mapper. Smart Learning Environments, 3(1), 10.

[11] M. T. Chi, and R. Wylie. 2014. The ICAP framework: Linking cognitive engagement to active learning outcomes. Educational Psychologist, 49(4), 219-243.

[12] C. A. Conkey, C. Bowers, J. Cannon Bowers, and A. Sanchez. 2013. Machinima and Video- Based Soft-Skills Training for Frontline Healthcare Workers. Games for health, 2(1), 39-43.

[13] M. W. Cronin, and K. A. Cronin. 1992. Recent empirical studies of the pedagogical effects of interactive video instruction in "soft skill" areas. Computing in Higher Education, 3(2), 53.

[14] F. D. Davis. 1989. Perceived usefulness, perceived ease of use, and user acceptance of information technology. MIS quarterly, 319-340.

[15] V. Dimitrova, and P. Brna. 2016. From Interactive Open Learner Modelling to Intelligent Mentoring: STyLE-OLM and Beyond. Artificial Intelligence in Education, 26(1), 332-349.

[16] E. Frias-Martinez, S. Y. Chen, R. D. Macredie, and X. Liu. 2007. The role of human factors in stereotyping behavior and perception of digital library users: a robust clustering approach. User Modeling and User-Adapted Interaction, 17(3), 305-337.
[17] M. Giannakos, D. Sampson, and Ł. Kidziński, 2016. Introduction to smart learning analytics: foundations and developments in video-based learning. Smart Learning Environments, 3(1), 1-9.

[18] P. J. Guo, J. Kim, and R. Rubin. 2014. How Video Production Affects Student Engagement: An Empirical Study of MOOC Videos. Proc. $1^{\text {st }}$ ACM Conf. Learning at Scale. 41-50.

[19] Y. Hara, Y. Tomomune, and M. Shigemori. 2004. Categorization of Japanese TV viewers based on program genres they watch. User Modeling and User-Adapted Interaction, 14, 87-117.

[20] S. G. Hart. 2006. NASA-task load index (NASA-TLX); 20 years later. Proc. the Human Factors and Ergonomics Society annual meeting, 50(9), Sage Publications. 904-908.

[21] H. U. Hoppe, M. Müller, A. Alissandrakis, M. Milrad, C. Schneegass, and N. Malzahn. 2016. "VC/DC"-Video versus Domain Concepts in Comments to Learner-generated Science Videos. In 24th Int. Conf. on Computers in Education. AsiaPacific Society for Computers in Education. 172-181.

[22] A. Jameson, B. Berendt, S. Gabrielli, F. Cena, C. Gena, F. Vernero, and K. Reinecke. 2014. Choice architecture for human-computer interaction. Foundations and Trends ${ }^{\circledR}$ in Human-Computer Interaction, 7(1-2), 1-235.

[23] G. R. Johnson. 1991. Teaching Tips for Users of the Motivated Strategies for Learning Questionnaire (MSLQ).

[24] A. Kleftodimos and G. Evangelidis. 2016. Using open source technologies and open internet resources for building an interactive video based learning environment that supports learning analytics. Smart Learning Environments, 3(1), 1-23.

[25] K. R. Koedinger, J. Kim, J. Z. Jia, E. A. McLaughlin, and N. L. Bier. 2015. Learning is not a spectator sport: Doing is better than watching for learning from a MOOC. Proc. $2^{\text {nd }} A C M$ Conference on Learning@ Scale. ACM. 111-120.

[26] G. Kovacs. 2016. Effects of in-video quizzes on MOOC lecture viewing, Proc. $3^{\text {rd }}$ Learning @ Scale, 31-40.

[27] M. Kravčík, and R. Klamma. 2011. On psychological aspects of learning environments design. In European Conference on Technology Enhanced Learning. Springer. 436-441.

[28] G. Kurtz, A. Tsimerman, and O. Steiner-Lavi. 2014. The Flipped-Classroom Approach: The Answer to Future Learning? European J. of Open, Distance and E-learning, 17(2), 172-182.

[29] Y. Long and V. Aleven. 2017. Enhancing learning outcomes through self-regulated learning support with an Open Learner Model. User Modeling and User-Adapted Interaction, 1-34.

[30] A. Louis and A. Nenkova. 2013. What Makes Writing Great? First Experiments on Article Quality Prediction in the Science Journalism Domain. Transactions of the Association for Computational Linguistics, 1, 341-352.

[31] J. Masthoff and J. Vassileva. 2015. Tutorial on Personalization for Behaviour Change. In Proceedings of the 20th International Conference on Intelligent User Interfaces (IUI '15). ACM. 439-442.

[32] L. N. Michaud, and K. F. McCoy. 2004. Empirical derivation of a sequence of user stereotypes for language learning. User modeling and user-adapted interaction, 14(4), 317-350.

[33] S. Michie, M. M. van Stralen, and R. West. 2011. The behaviour change wheel: a new method for characterising and 
designing behaviour change interventions. Implementation science, 6(1), 42.

[34] A. Mitrovic, V. Dimitrova, A. Weerasinghe, and L. Lau. 2016. Reflexive experiential learning using active video watching for soft skills training. Proc. $24^{\text {th }}$ Int. Conf. Computers in Education. APSCE. 192-201.

[35] A. Mitrovic, V. Dimitrova, L. Lau, A. Weerasinghe, and M. Mathews. 2017. Supporting constructive video-based learning: requirements elicitation from exploratory studies. (accepted for $19^{\text {th }}$ Int. Conf. on Artificial Intelligence in Education -AIED 2017).

[36] R. Münscher, M. Vetter, and T. Scheuerle. 2015. A review and taxonomy of choice architecture techniques. Journal of Behavioral Decision Making, 29, 511-524.

[37] G. Paliouras, V. Karkaletsis, C. Papatheodorou, and C. D. Spyropoulos. 1999. Exploiting learning techniques for the acquisition of user stereotypes and communities. In UM99 User Modeling. Springer Vienna. 169-178.

[38] A. Pardo, N. Mirriahi, S. Dawson, Y. Zhao, A. Zhao, and D. Gaševic. 2015. Identifying learning strategies associated with active use of video annotation software. Proc. 5th Int. Conf. Learning Analytics and Knowledge. ACM. 255-259.

[39] Pew Research Centre. 2015. 5 facts about online video, for YouTube's 10th birthday, Report, January 2015.

[40] P. R. Pintrich, and E. V. De Groot. 1990. Motivational and self-regulated learning components of classroom academic performance. Journal of educational psychology, 82(1), 33.

[41] R. A. Sottilare, A. Graesser, X. Hu, and B. Goldberg. (Eds.). 2014. Design Recommendations for Intelligent Tutoring
Systems: Volume 2-Instructional Management (Vol. 2). US Army Research Laboratory.

[42] T. Stajner, B. Thomee, A.M.Popescu, M.Pennacchiotti, and A. Jaimes. 2013. Automatic selection of social media responses to news. Proc. 19th ACM SIGKDD international conference on Knowledge discovery and data mining. ACM. 50-58

[43] C. Tan, L. Lee, and B. Pang. 2014. The effect of wording on message propagation: Topic- and author-controlled natural experiments on Twitter. Proc. ACL 2014.

[44] A. Tatar, P. Antoniadis, M. D. De Amorim, and S. Fdida. 2014. From popularity prediction to ranking online news. Social Network Analysis and Mining, 4(1), 1-12.

[45] D. Thakker, D. Despotakis, V. Dimitrova, L. Lau, and P. Brna. 2012. Taming digital traces for informal learning: a semanticdriven approach. In European Conf. Technology Enhanced Learning. Springer. 348-362.

[46] R. H. Thaler, and C. R. Sunstein. 2008. Nudge: Improving Decisions about Health, Wealth, and Happiness. Yale University Press, New Haven.

[47] I. Vieira, A. P. Lopes, and F. Soares. 2014. The potential benefits of using videos in higher education. Proc. EDULEARN14 Conf. (pp. 0750-0756). IATED Publications.

[48] J. Wachtler, M. Hubmann, H. Zöhrer, and M. Ebner. 2016. An analysis of the use and effect of questions in interactive learning-videos. Smart Learning Environments, 3(1), 13.

[49] A. M. F. Yousef, M. A. Chatti, and U. Schroeder. 2014. The state of video-based learning: A review and future perspectives. Int. J. Adv. Life Sci, 6(3/4), 122-135. 